Recepción: 24 / 09 / 2018

Aceptación: 16 / 10 / 2018

Publicación: 03 / 12 / 2018

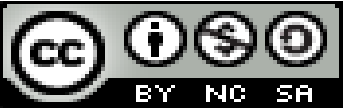

Ciencias económicas y empresariales

Artículo de Investigación

\title{
Análisis del impacto publicitario en la decisión de compra en empresas del sector comercial
}
Analysis of the advertising impact in the decision of purchase in business companies

\section{Análise do impacto publicitário na decisão de compra em empresas de negócios}

\author{
Ana M. Quezada-Ureña ${ }^{\mathrm{I}}$ \\ aquezadaanita0639@uta.edu.ec \\ Leonardo G. Ballesteros-López II \\ leonardogballesteros@uta.edu.ec \\ Cesar A. Guerrero-Velastegui III \\ ca.guerrero@uta.edu.ec \\ Edwin J. Santamaría-Freire IV \\ edwinjsantamaria@uta.edu.ec
}

Correspondencia: aquezadaanita0639@uta.edu.ec

\footnotetext{
I Docente de la Universidad Técnica de Ambato, Ambato, Ecuador.

II Magíster en Gestión Estratégica Empresarial MBA, Ingeniero Comercial, Licenciado en ciencias Administrativas Administrador en Mercadotecnia, Docente de la Universidad Técnica de Ambato, Ambato, Ecuador.

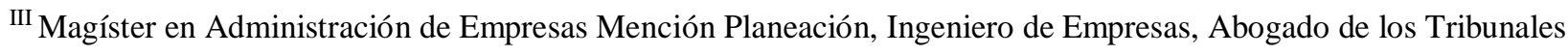
y Juzgados de la República del Ecuador, Docente de la Universidad Técnica de Ambato, Ambato, Ecuador.

${ }^{\text {IV }}$ Magíster en Administración, Máster Universitario en Estadística Aplicada, Ingeniero en Administración de Empresas y Negocios, Docente de la Universidad Técnica de Ambato, Ambato, Ecuador.
} 


\section{Resumen}

El impacto que la publicidad tiene hoy en día tiene una significancia ya que se reciben alrededor de 3.000 a 5.000 impactos publicitarios, diariamente, las estrategias que el marketing ofrece, permiten determinar las siguientes características, por qué, qué, cómo, dónde, cuándo y a quién deben llegar estos mensajes. Las innumerables luchas publicitarias se libran en el corazón, para crear una corriente de simpatía, un vínculo duradero con los públicos, esta es la finalidad de los anuncios y de toda publicidad que se aprecia. En otras palabras, es evidente, el fin que tiene la comunicación empresarial, como lo es mover a la acción, crear vínculos emocionales entre la marca y sus públicos que propicien una relación duradera. El objetivo de esta investigación se determina el alcance que tiene el impacto publicitario, en la decisión de compra en empresas comerciales, en la actualidad, entendiéndose las variables del estudio, por esta razón permite al usuario desde la consideración de una necesidad hasta la compra de un producto o servicio, por otra parte, la publicidad como parte de un programa de marketing para aumentar las ventas de los productos o servicios. La metodología de esta investigación, fue de campo, lo cual permitió obtener óptimos resultados para el desarrollo del estudio, así como las técnicas a usar fue de encuestar, estuvo dirigida a las empresas comerciales aledañas a la provincia de Tungurahua, pudiendo evaluar el impacto que genera su publicidad, en la decisión de la compra para los consumidores, como resultado se logró un alcance verídico, sobre aquellas empresas comerciales que tienen como estrategia principal el marketing de la publicidad digital. Adicionalmente se puede concluir que por medio de esta investigación podemos visualizar cuales son las necesidades y deseos de los consumidores por otra parte analizar la publicidad en empresas comerciales y saber qué impacto llega a sus clientes y de esa manera ver si les beneficia para la venta de su producto o servicio.

Palabras clave: publicidad; servicio; empresas comerciales; marketing digital.

\section{Abstract}

The impact that advertising has today has a significance as they receive around 3,000 to 5,000 advertising impacts, daily, the marketing strategies offered, allow us to determine the following characteristics, why, what, how, where, when who should get these messages. The innumerable advertising fights are fought in the heart, to create a current of sympathy, a lasting bond with the public; this is the purpose of the advertisements and of all the publicity that is appreciated. In 
other words, it is evident, the purpose of business communication, as it is to move to action, to create emotional links between the brand and its public that foster a lasting relationship. The objective of this research is to determine the scope of the advertising impact, in the purchase decision in commercial companies, at present, understanding the variables of the study, for this reason it allows the user from the consideration of a need until the purchase of a product or service, on the other hand advertising as part of a marketing program to increase sales of products or services. The methodology of this research was field, which allowed to obtain optimal results for the development of the study, as well as the techniques to be used was to survey, was directed to the commercial companies surrounding the province of Tungurahua, being able to evaluate the impact that generates its publicity, in the decision of the purchase for the consumers, as a result a true reach was achieved, on those commercial companies whose main strategy is the marketing of digital advertising. Additionally, it can be concluded that through this research we can visualize what the needs and desires of consumers are, on the other hand, analyze the advertising in commercial companies and know what impact it reaches their clients and in this way see if it benefits them for the sale of your product or service.

Key words: advertising; service; trading companies; digital marketing.

\section{Resumo}

O impacto que a publicidade tem hoje tem um significado já que cerca de 3.000 a 5.000 impactos de publicidade, estratégias diárias que o marketing de propostas recebidas, para a determinação das seguintes características, por isso, o quê, como, onde, quando e quem deve receber essas mensagens. Publicidade inúmeras lutas travadas no coração, criando uma onda de simpatia, um vínculo duradouro com o público, este é o propósito de anúncios e publicidade tudo apreciado. Em outras palavras, é evidente, o objetivo da comunicação empresarial, como é agir, criar laços emocionais entre a marca e seu público que promovem um relacionamento duradouro. O objetivo deste escopo pesquisa tem o impacto da publicidade nas decisões de compra em empresas comerciais, no momento, o que significa que as variáveis do estudo, portanto, permite que o usuário da conta de uma necessidade de comprar é determinado um produto ou serviço, por outro lado, publicidade como parte de um programa de marketing para aumentar as vendas de produtos ou serviços. A metodologia desta pesquisa foi de campo, o que permitiu que os resultados ideais 
para o desenvolvimento de estudos e técnicas para usar foi vistoriado, visava vizinhos empresas comerciais para a província de Tungurahua, sendo capaz de avaliar o impacto Gera sua publicidade, na decisão da compra para os consumidores, como resultado de um verdadeiro alcance alcançado, nas empresas comerciais cuja principal estratégia é a comercialização da publicidade digital. Além disso pode-se concluir que, através desta pesquisa, podemos ver quais são as necessidades e desejos dos consumidores, por outro lado analisar as empresas de publicidade de negócios e saber qual o impacto que atinge seus clientes e, assim, ver se ele beneficia-los para vender seu produto ou serviço.

Palavras chave: publicidade; serviço; empresas de negócios; marketing digital.

\section{Introducción}

Para poder comprender de mejor manera cómo influye el impacto publicitario, en un consumidor final, o futuro comprador hay que tomar en cuenta lo que el autor, (Luis Bassat, 2016), indica en su (Libro rojo de la Publicidad) que la "La publicidad es el arte de convencer consumidores", debido a que las disciplinas del marketing posee un fundamento basado en la proporción entre ciencia y arte, a su vez, indica este mismo autor que la publicidad es un puente que se encuentra entre el producto y servicio.

Mientras que (Bill Berbanch, 2013), afirma que "La publicidad es fundamentalmente persuasión, y la persuasión no es una ciencia, es un arte”. Cabe destacar que (Philip Kotler, 2013), manifiesta que la publicidad es “Como una comunicación no personal y onerosa de promoción de ideas, bienes o servicios, que lleva a cabo un patrocinador identificado". En la actualidad, existe mucha polémica acerca de cómo interactúa la eficacia publicitaria, debido a la gran cantidad de mensajes, dentro de los cuales se ve expuesto el comprador diariamente, es necesario adoptar la teoría del (Philip Kotler, 2013) quien fue uno de los expertos más destacados en la práctica estratégica del marketing, manifiesta que el sujeto reconoce la necesidad, y a través de esta identifica su insatisfacción y esto lo conlleva en la búsqueda de lo que desea conseguir, esto es posible por el estímulo de estas necesidades, las cuales se consiguen por medio de anuncios publicitarios, escaparates, la publicidad en el lugar de venta, entre otros, de esta misma manera este autor sostiene que el consumidor tiende a buscar información, de manera pasiva o activa. 
De lo anterior expuesto, cabe resaltar que primeramente el consumidor selecciona su publicidad esto es de acuerdo al promedio, que tiene mayor exposición a la misma, por las cuales debe pasar el consumidor, se le puede considerar como más de un millar de artículos publicitarios, dentro de los cuales un consumidor normal, siempre recordara con precisión las tres publicidades que más llamaron su atención, mientras que los restantes luchan por encabezar este ranking sin escatimar en sus esfuerzos creativos, es importante mencionar al Ing. (Olsztyn Mario, 2018), el cual cerciora que la conducta de compra, está íntimamente relacionada con factores principales de la esencia en la vida del individuo y el medio social en el que actúa, como ya se ha venido desarrollando surge de una necesidad.

Como ya se ha manifestado, en el individuo influye siempre factores que motivaran al consumidor, a finalizar su necesidad con la compra que fue dirigida por medio de un aviso publicitario, en cualquiera de sus dimensiones, como lo indican (Bello, Vázquez y Trespalacios, 2016), la importancia sobre una correcta definición de objetivos publicitarios se hace manifiesto a la hora de medir la eficacia publicitaria., ya que los objetivos son indispensables para poder realizar estudios de eficacia, esto hace referencia a que toda campaña publicitaria, debe ir de la mano con los objetivos para que se pueda dar la decisión de compra, mientras tanto (Schultz, Martin y Brown, 2014), "distinguen tres enfoques diferentes a la hora de fijar los objetivos de una campaña publicitaria" en función de las ventas, en términos de conducta o basados en los efectos de la comunicación (Bello, Vázquez y Trespalacios, 2016).

Para detectar, la problemática de esta investigación es necesario tomar en cuenta ciertos criterios que han traído un crecimiento significativo en la toma de decisiones dentro del impacto publicitario. Esto se debe a que gracias a la evolución que ha tenido la tecnología en la publicidad, se ha tornado en un giro de $180^{\circ}$, alargando sus horizontes, cada vez más creativos, incursionando al mundo cibernético, por lo que ha ofrecido cada vez más una mayor visibilidad de lo que una empresa persigue, en transmitir en "sus mensajes o avisos publicitarios". Esto ha sido comprobado en numerosas investigaciones como lo que la publicidad que más gusta vende más, de acuerdo al autor ya antes mencionado, (Luis Bassat, 2016), "una simple escala de los spots permitía adivinar el 87\% de las compras, por lo tanto, la relación que existía entre "gustar" y "comprar" era realmente grande" 
Por lo que hoy en día la publicidad, se entiende como el medio de comunicación, que conduce a una idea o mensaje sobre una determinada marca o tema determinado, encontrando sus nuevas herramientas, como grandes oportunidades para crear la credibilidad entre la audiencia y atraer día a día nuevos consumidores. Es de esta manera cómo surge, la hipótesis de (González, 2014), quien “denota la existencia de una discordancia entre lo percibido por las organizaciones con lo que aprecia el cliente". Esto suele ser grave por lo general ya que para que se dé una buena relación con el consumidor y la publicidad, debe estar claramente definido el objetivo que persigue la publicidad, pese a que, si no se está transmitiendo la información de una manera clara, entonces se está perdiendo clientes y nuevos consumidores.

Cada una de las teorías planteadas, son importantes para establecer, un buen servicio por parte de las empresas comerciales, debido a que es importante identificar, que factores más influyen, en el área de marketing y adoptarlos como aliados gerenciales, los mismos inciden, en la calidad del servicio, el bienestar y un buen desarrollo, con la única finalidad de optimizar las operaciones, que sean principales activos dentro de las empresas comerciales, está se encuentra representado por los usuarios o consumidores, cuando se trata de prestar un servicio, así lo expresa (Doris Martínez, 2016).

Esto resulta conveniente y saludable, para los accionistas o gerentes en empresas, el detenerse y poder autoanalizarse, de cierta manera en un periodo definido sobre su desempeño, por lo que la misma autora, hace mención como un concejo sobre la revisión o auditoria empleada de manera periódica y regular, dado a su carácter preventivo, y de esta manera evitar que los cambios futuros del entorno, sorprenda de manera progresiva como desprevenidos, y así mejorar su impacto publicitario, sin dejar de seguir en el actual auge de la publicidad y en el marketing digital.

El objetivo de esta investigación es determinar el alcance que tiene el impacto publicitario, en la decisión de compra en empresas comerciales, en la actualidad, es de esta manera como se ha planteado, esta investigación por lo que la pregunta de la investigación, radica en cuál ¿Hoy en día estaríamos en frente de un impacto publicitario eficaz, y que actividades están persiguiendo las empresas para proveer la necesidad del consumidor en la decisión de su compra? 
Esta investigación, surgió con la finalidad comprender si realmente existe un impacto publicitario en nuestros días, es decir si realmente resulta interesante al consumidor la publicidad que estas empresas lanzan al mercado, es por ello que para determinar esta hipótesis es necesario tomar en cuenta, otras investigaciones que proporcionaran información de relevancia, para esta investigación.

Para ello se tomó en cuenta, a la autora, la cual realizo artículo científico, titulado: Factores clave en marketing enfoque: empresas de servicios. Orbis. Revista Científica Ciencias Humanas, la misma se llevó a cabo en la ciudad de Maracaibo, Venezuela.

“El principal objetivo, a identificar, dentro del artículo científico, radica en cuáles eran los factores más claves, en el éxito del área de marketing, a dentro de los mismos, serán definidos como aliados gerenciales muy importantes, para las empresas que persigan el posicionarse dentro del mercado, y a su vez bajo un buen desempeño totalmente sostenible, es por ello, que, por medio de consultas, con referencias teóricas del Marketing como aliado gerencial, con recopilación e integración de datos, permitió encontrar como hallazgos, específicamente quince herramientas de apoyo, comprendidas como prevención y control de los llamados "factores clave de éxito (FCE)" dentro del área de marketing, como aliados gerenciales de las empresas de servicios en el sector seguridad, vigilancia y protección en los sectores industriales, comerciales y residenciales” (Doris Martínez, 2016)

De esta manera, se puede observar que las variables de este estudio se encuentran comprendidas, en esta investigación, antes señalada, por lo que determino que aquello que más influye para el éxito de un buen marketing, es la prevención y control, para el llamado factores claves del éxito, de esta manera la autora, determino que es importante que para aquellos gerentes o socios a cargo de las empresas, es imprescindible la evaluación de su mercadeo, y publicidad, para no pormenorizar el objetivo de su publicidad, (Doris Martínez, 2016), en comparación con la investigación del Ing. Comercial, titulado de la siguiente manera Análisis del comportamiento del consumidor en la compra de tablets en la ciudad de quito, para personas entre los 18 a 40 años de edad, en el año 2013. 
“Como fundamento importante para la decisión de compra se considera, el comportamiento del consumidor comprende básicamente en conocer, cuáles son las cualidades que motivan a los consumidores en adquirir cierto bien o servicio, para el autor el análisis del comportamiento del consumidor de tablets en Quito era un tema sumamente nuevo, que no había sido tratado, hasta el momento ya que estos dispositivos son relativamente nuevos en el mercado actual, y por este mismo motivo es importante realizar un estudio para determinar cuáles son los gustos y preferencias de los usuarios de tabletas, y este estudio arrojo como resultado se determinó que los principales motivos para la compra de tablets, eran la funcionalidad y la practicidad del producto, ya que por lo general, se puede ocupar una Tablet" (Pablo Arcos, 2014).

Para los consumidores finales de estas Tables, era importante las actividades que le permitiría la realización de tareas cotidianas del trabajo o estudio ya que su tamaño le permite se trasportada para mantenerse informado la mayor parte del tiempo, cabe resaltar que el estudio permitió entender que aquello que más influye en la decisión de su compra, es la manera en cómo la presenta, esto quiere decir que, para ellos era atractivo, las aplicaciones, así como el modelo que la publicidad, enseñaba, así lo expreso este autor (Pablo Arcos, 2014).

Como referencias bibliografías de este estudio, se tomó en cuenta estas investigaciones y artículos, ya que, por su complejidad, permitió orientar esta investigación hacia un grado de más profundidad, ya que no solo basta que influya la publicidad si no que esta debe persuadir en todos sus sentidos al consumidor, aquello que más llama la atención del individuo es la Calidad del producto, ya que un buen producto siempre atrae nuevos clientes y afianza a los que ya han comprado.

Cabe destacar que la opinión de (Deming, E, 2018), quien afirma que la calidad es "un grado predecible de uniformidad y fiabilidad a bajo coste, adecuado a las necesidades del mercado". El autor así mismo, indica que uno de los principales objetivos de la empresa debe ser permanecer en el mercado, y así lograr proteger la inversión, para poder ganar dividendos y asegurar los empleos. Para (Juran y Gryna, 2010), la calidad lo define como la adecuación al uso, ya que implica una adecuación en el diseño del producto, mayor aun en el servicio destacado como la calidad del diseño y por ende la medición del grado en donde el producto es realizado conforme, al dicho diseño determinado como la calidad de fabricación o conformidad. 
Es por ello que logran influenciar tanto en el consumidor, quien examinara si le es conveniente, todas las características que ofrece el producto por medio del impacto publicitario que genera, y es allí donde él se pregunta si realmente, al adquirir el mismo, estaría frente a la solvencia completa o parcial de su necesidad.

La relación, que guarda la variable de decisión de compras, en el impacto de la publicidad, indudablemente es inseparable ya que no se puede dar ningún caso sin la complementación de la otra, como sugerencias de estudios similares es necesario, mencionar esta investigación, la cual estudia ambas variables y es titulada de la siguiente manera; análisis del impacto de la publicidad en las decisiones de consumo en los niños. Esta hace referencia al rol que cumple la publicidad y el marketing, se considera que esta jugada es totalmente importante, para manipular de forma directa la conducta, acerca de las mismas, puesto que se centran en la atención hacia los grupos humanos más susceptibles, dentro de los cuales están los niños, no es un secreto que en esa etapa de desarrollo aprenden más rápido y por tanto consideran, que lo que les puede brindar el uso de los productos promocionados es la codiciada inserción social, por ello se dice que actualmente más del 95\% de los hogares, sin tomar en cuenta que el estatus social y económico, posee al menos un televisor, esto no impide que los niños, puedan escaparse de su atractivo, estudios recientes han permitido indagar que dentro de las familias argentinas, al momentos de ir de compras, los niños logran abarcar, en promedio, aproximadamente un 25\% dentro de lo que es el presupuesto familiar. (Sonia Ottaviano, 2015)

Como segunda referencia de ambas variables, encontramos la siguiente, la toma de decisiones en el mundo del consumo basado en procesos cerebrales, la cual establece, que es importante para lograr establecer las marcas y las empresas, siempre y cuando se tenga en cuenta, la manera en cómo se desarrolla el mundo de la publicidad, y cómo cambian las acciones de los consumidores ante dichos desarrollos, ya que con el paso de los años, los expertos publicitarios, han podido notar que no hay como poder conocer cuál será la reacción de los consumidores ante una cierta comunicación publicitaria, como bien se sabe cada consumidor, tiene una cierta manera de actuar a la hora de tomar una decisión en el mundo del consumo, y por esta razón es que hay que investigar, cuales son los procesos, mediante el cual el consumidor llega a tomar una decisión. (María Tobar, 2013). 
Es importante destacar que los procesos cerebrales para el manejo del impacto publicitario, se ha desarrollado tan rápidamente esto se debe a que hoy en día es el más común lanzado por empresas, conocido como neuromarketing, esta se comprende como la aplicación de ciertas técnicas de la neurociencia al marketing, la cual busca conocer y comprender con los niveles, que demuestre en atención a las personas, estas son con diferentes estímulos, es así como se trata de explicar el comportamiento de las personas desde la base de su actividad neuronal.

Esta comprende el proceso para la decisión de comprar, es una representación por la cual pasa el consumidor, derivada de distintos periodos previos, esta surge cuando se reconoce que existe una necesidad, que para el consumidor se convierte en el problema, de una determinada adquisición de un bien o "compras", seguidamente el consumidor emplea todas las búsquedas posibles para determinar la información necesaria del producto, es a través de un aviso publicitario, por lo general las personas toman en cuenta las referencias que se hacen sobre el producto, es aquí cuando comienza la batalla entre las demás publicidades, por lo que suele ganar aquella que más cubra las necesidades que manifieste el futuro comprador.

La buena publicidad forma e informa al consumidor, esto comprender la publicidad, en la manera en cómo se ha revelado como uno de los instrumentos efectivos de formación y prevención del consumidor. (Luis Bassat, 2016)

Algunas empresas, han hecho mención en que las decisiones de compras, ya que se basan en ser emocionales, por lo que estas decisiones tienen un soporte racional, esto puede comprenderse por que los seres humanos, en la toma de decisiones no bastan con tomar la primera que pase por su mente, sino que en su búsqueda trata de adoptar aquella que más convenga, y es de saberse que no cuenta con una sola sino con varias, (Abraham Geifman, 2014), hace mención sobre un artículo publicitario más consistentes y exitoso en México, es la del almacén departamental "El Palacio de Hierro", bajo el slogan: "Soy totalmente palacio".

Esta apela de manera directa, a una introspección de la mujer, que no tiene nada de racional. Sin hablar de precios, variedad, calidad u otros desgastados argumentos, ha penetrado en la mente de las consumidoras y ha generado un posicionamiento sólido e inamovible, otra referencia importante la hace el autor que se menciona a continuación, puesto que hace referencia en como una persona comienza la decisión de su compra desde la etapa inicial, conocida como la 
necesidad hasta la etapa final, cuando compra el producto, porque el mismo determino que eran ideales para su necesidad, esto ya una vez que evaluó la publicidad.

"Un ejemplo claro se basa en la compra de un nuevo teléfono inteligente, para esta compra, es probable que la necesidad del usuario, sea que necesite comunicación o acceso a Internet, o un problema, ya que no puede interactuar con amigos a través de las redes sociales. El valor agregado de productos como Android, iPhone o Windows Phone y otros deben satisfacer su necesidad o resolver su problema. En segundo lugar, el consumidor desea hablar con sus amigos y, por lo tanto, navegar por Internet. Ya que como comprador, puede visitar una tienda local de teléfonos celulares y hablar con el personal de ventas para ayudarlo a completar la tercera etapa, es decir, su evaluación de alternativas, la última etapa consiste en la selección del producto, donde toma la decisión final y comprar Su teléfono inteligente en una tienda local o a través de un sitio web de comercio electrónico, es cómo básicamente las personas hacen sus compras teniendo en cuenta, que toman la decisión de la que mejor se adapte a sus necesidades” (Kotler, Armstrong, 2018).

Dentro de las propiedades y características de la variable definida, se puede añadir lo que aporta (Webloyalty y Oxford Brookes University, 2017), en recientes estudios que se realizaron el universidad Oxford, establecieron que son 5 los motivos que impulsan a los consumidores a tomar la decisión de compra de un determinado producto, el primero es el precio, el segundo la funcionalidad, el tercero los beneficios, y por ultimo las opiniones de otros usuarios y la personalización, es desde este punto que deben partir los impactos publicitarios, no solo crear una campaña se debe estudiar cómo llegar a la mente, del consumidor final, es por ello que se para poder completar el desarrollo esta premisa, se detallara la segunda variable de este artículo científico.

Según (O'Guinn, Allen y Semenik, 2016), estos autores del libro "Publicidad", definen la misma como "La publicidad es un esfuerzo pagado, trasmitido por medios masivos de información con objeto de persuadir", mientras que (American Marketing Asociation, 2016), dice que consiste en "la colocación de avisos y mensajes con finalidad persuasivos, en tiempo o espacio, adquirido por diferentes métodos y medios de comunicación por empresas lucrativas, organizaciones no lucrativas, agencias del estado y los individuos que intentan informar y/o persuadir al igual que los miembros de un mercado meta en particular o a audiencias acerca de sus productos, servicios, organizaciones o ideas". 
Es de saber que la publicidad adoptada por empresas comerciales, es una forma de comunicación imprecisa y de largo alcance, la misma es cancelada por un patrocinador en este caso, una empresa que busca las compras de nuevos y viejos clientes, para así aumentar sus ingresos monetarios, y convertirse en una marca, o en los proveedores de servicios más sofisticados de la sociedad.

Dentro del impacto publicitario en empresas comerciales, un estudio reciente de la Universidad Técnica de Ambato, realizada por (Jenny Camino, 2014), como "Estrategias de publicidad y su impacto en las ventas de la empresa Repremarva de la ciudad de Ambato, durante el año 2012", los resultados más resaltantes de esta investigación radican en una encuesta dirigida a los clientes de la empresa estudiada, dentro de los cuales supieron manifestar que el $91.1 \%$ se encuentran totalmente de acuerdo, en la aplicación de nuevas estrategias publicitarias, porque estas son necesarias para el desarrollo del volumen de ventas, y nuevos consumidores, esto permitió presentarse como un factor determinante que instigue a la alta dirección de la entidad a encontrar la estrategia precisa que ayude y resalte la imagen empresarial.

Es muy importante que las empresas escuchen y pongan en prácticas aquellas nuevas tendencias y técnicas que sugieran sus clientes, puesto a que el cliente es el que gana más publicidad incluso, es este quien por medio de recomendaciones hace crecer en el mercado la demanda de los usuarios. Las propiedades y características más relevantes del impacto publicitario son, la publicidades hechas por empresas deben estar hecha de manera clara, es decir no debe tener nada confuso, ni nada que le haga cambiar de parecer al consumidor, los anuncios deben generar una respuesta inmediata, es decir que al observar las publicidades por las empresas los usuarios, sientan la necesidad de adquirirlo inmediatamente y de esa manera demostrarle que el producto, es todo lo que necesita el cliente, siempre deben estar presentes en todos lados es decir, en cada medio publicitario que le permitan dar a conocer sus servicio y productos.

\section{Materiales y Métodos}

Para la presente investigación, se procedieron a seleccionar los materiales y métodos, idóneos, los cuales serán descritos a continuación. Para (Kuhn, 1975), se dice que un paradigma existe cuando, se asocia al campo de la investigación, cuando este ha podido conquistar, ciertos espacios connotativos, que se sitúan dentro de un lugar privilegiado, en resumidas palabras es la 
filosofía de la investigación, a su vez, (Grinnell, 2006) señala que existen dos enfoques cualitativo, y cuantitativo, que forman parte de la investigación científica, ya que ambos se emplean de la misma manera cuidadosa y sistemáticamente, por lo tanto ambos enfoques fueron acogidos en esta investigación, la cualitativa ya que se encargó del estudio del fenómeno dentro su área natural, y la cuantitativa, se encargó de reunir los datos numéricos, de la encuesta aplicada, en lo que concierne al tipo de investigación, para (Tamayo, M, 2016), se propone la descripción de modo sistemático, en base a las características de una población, situación o área de interés.

La población, que fue objeto del estudio, dentro de esta investigación, se basa en un total de 385 personas, las cuales, fueron dirigidas a empresas comerciales para que de esa manera se da conocer el grado de decisión de compra y a su vez el a nivel de publicidad que hay en cada una de las empresas. Por lo cual fue tomado en cuenta de 9 cantones de la provincia de Tungurahua.

Tabla 1 Población del estudio

\begin{tabular}{ll}
\hline Cantón & Total \\
\hline Baños & 18 \\
Cevallos & 6 \\
Mocha & 5 \\
Patate & 11 \\
Pelileo & 44 \\
Píllaro & 28 \\
Quero & 15 \\
Tisaleo & 5 \\
Ambato & 253 \\
Total & $\mathbf{3 8 5}$ \\
\hline
\end{tabular}

\section{Elaborado por: Autores (2018)}

De acuerdo manifestado por (Moreno, E, 2013), un instrumento de investigación es la herramienta usada, con el fin de recoger información por parte del investigador, y así cuantificar las respuestas, para aportar una posible solución al problema investigado, de esta manera es necesario una vez que se seleccionaron los instrumentos, adecuados, hacer la respectiva 
validación del instrumentos, para medir el grado de confiabilidad, y poder medir el margen de error. Cabe destacar que, (Rojas, I, 2011, pág. 280), menciona que la validación, se basa en el procedimiento, donde se expone, un instrumento a través de ciertas de tácticas, tales como la consulta y prueba con expertos, calificando el instrumento, como él idóneo, recomendando modificaciones al diseño; y como segundo lugar, una prueba piloto del instrumento, donde es aplicado a personas, asemejándose a la población objeto o forman parte de ella. Tomándose en cuenta la encuesta, siendo uno de los instrumentos, más aplicados en investigaciones, y a su vez, brinda un grado de confiabilidad ya que son, los datos recabados de la misma población, donde se sitúa el problema de la investigación, para ello se dice que la encuesta, para (Pobea, M, 2015), es una técnica de recogida de datos, por medio de una aplicación de un cuestionario, a una determinada muestra.

Tabla 2 Variables y Dimensiones

\begin{tabular}{cl}
\hline \multicolumn{1}{c}{ VARIABLES } & \multicolumn{1}{c}{ DIMENSIONES } \\
\hline \multirow{2}{*}{ Decisión de compra } & Precio \\
& Funcionalidad \\
& Pensamientos de los usuarios \\
& Reacción positiva \\
Publicidad en empresas comerciales & Necesidad \\
& Adquisición del producto \\
\hline
\end{tabular}

Elaborado por: Autores (2018)

La encuesta fue aplicada, a 9 cantones de la provincia de Tungurahua, donde se pudo acceder directamente a los clientes de diferentes empresas comerciales, donde se les realizaron 8 preguntas, acerca de la decisión de compra, y la manera en como la publicidad influía, en sus decisiones, así mismo, se procedió a la recolección de las respuestas señaladas. En cuanto a la descripción, estadística se procedió a usar el programa estadístico de Excel, para la tabulación de la encuesta, siendo, un mecanismo confiable ya que el grado de confiabilidad, se basa en un $95 \%$, a su vez se procedió a realizar estadística descriptiva de los resultados, que serán analizados a continuación. 


\section{Resultados}

Como resultado final de las encuestas, se tomaron en cuenta preguntas en función de las variables del estudio, que fueron necesarias para medir el impacto publicitario que tienen, sobre la decisión de compra, en primer lugar, analizaremos lo señalado anteriormente con la aplicación estadística de chi cuadrado por lo que fue tomado en cuenta las variables y de acuerdo a sus 9 cantones de Tungurahua.

\section{Gráfico 1 Encuesta realizada a empresas comerciales}

\section{PERCEPCIÓN DE UN ANUNCIO PUBLICITARIO}

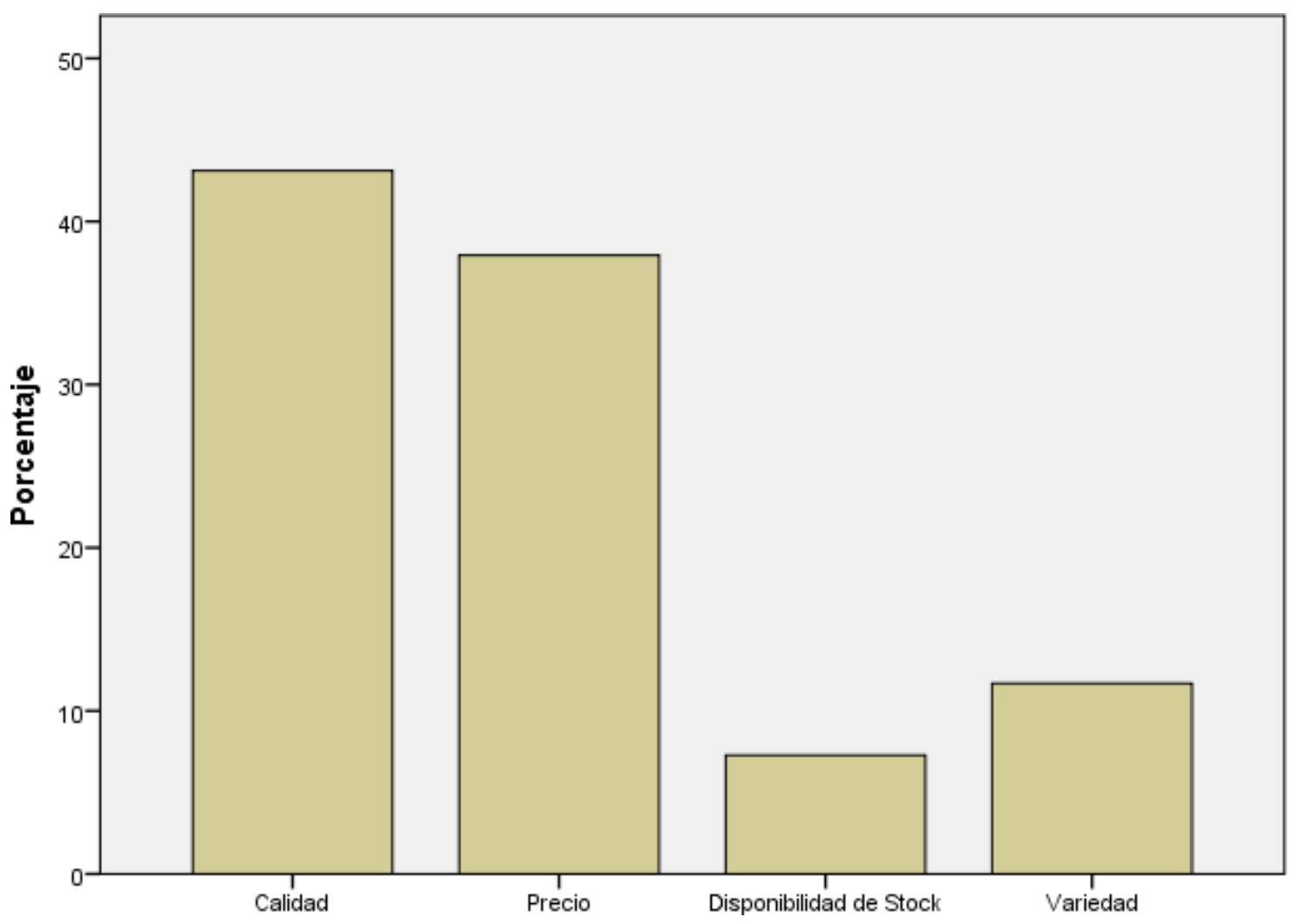

Elaborado por: Autores (2018)

De acuerdo a la encuesta realizada a los 9 cantones de la provincia de Tungurahua sobre las percepciones ante un anuncio publicitario sobresale con un máximo porcentaje la calidad de $43 \%$, con un $37.9 \%$ precio, $11 \%$ variedad y como un porcentaje menor de $7.3 \%$ que existe en la disponibilidad de stock. 


\section{Gráfico 2 Encuesta realizada a empresas comerciales}

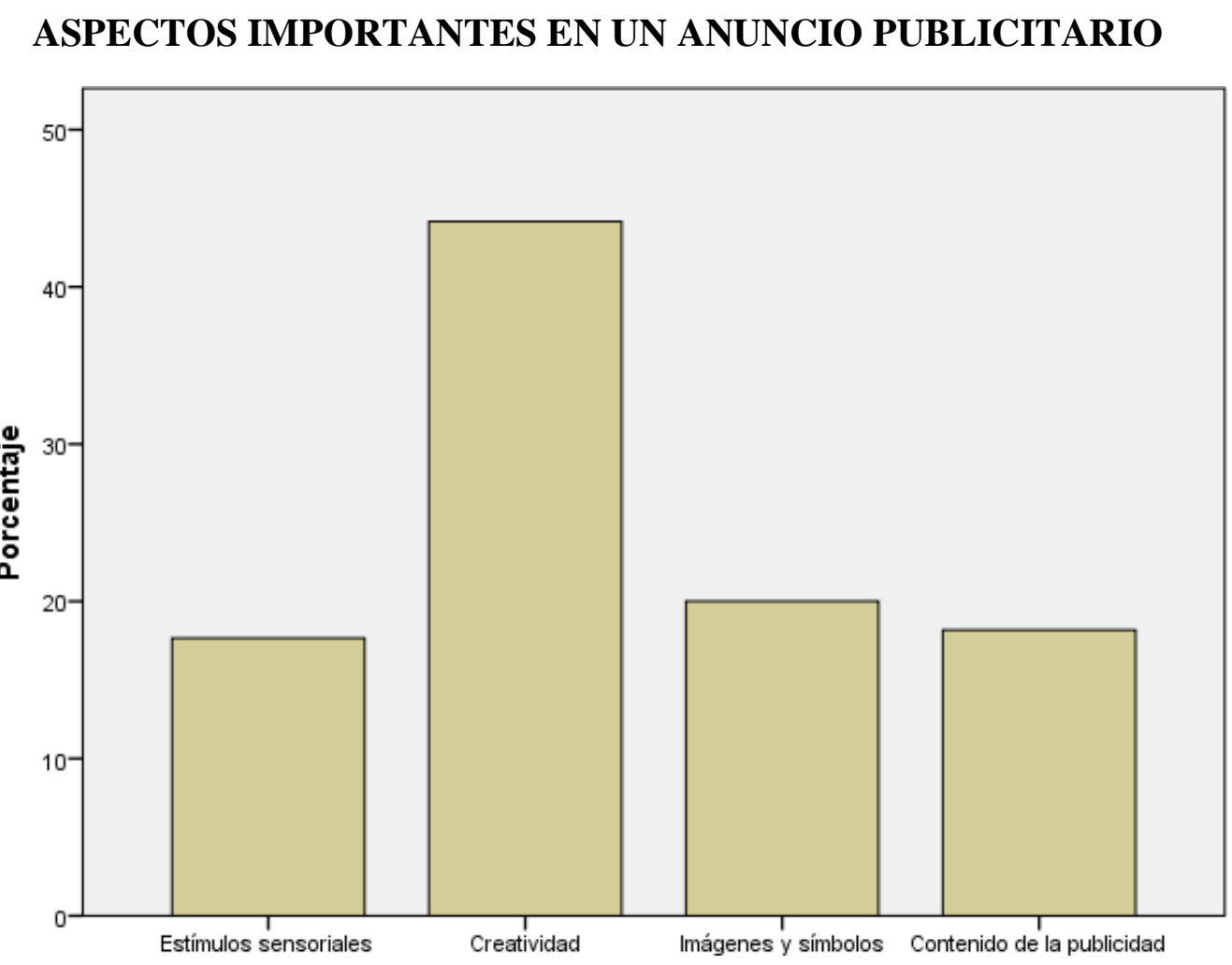

\section{Elaborado por: Autores (2018)}

De acuerdo a la encuesta realizada a los 9 cantones de la provincia de Tungurahua sobre los aspectos ante un anuncio publicitario sobresale con un máximo porcentaje la creatividad de $170 \%$, con un $77 \%$ imágenes y símbolos, $70 \%$ contenido de la publicidad y como un porcentaje menor existe los estímulos sensoriales. 


\section{Gráfico 3 Encuesta realizada a empresas comerciales}

\section{FACTORES DE LAS DECISIONES FINALES DE COMPRA}

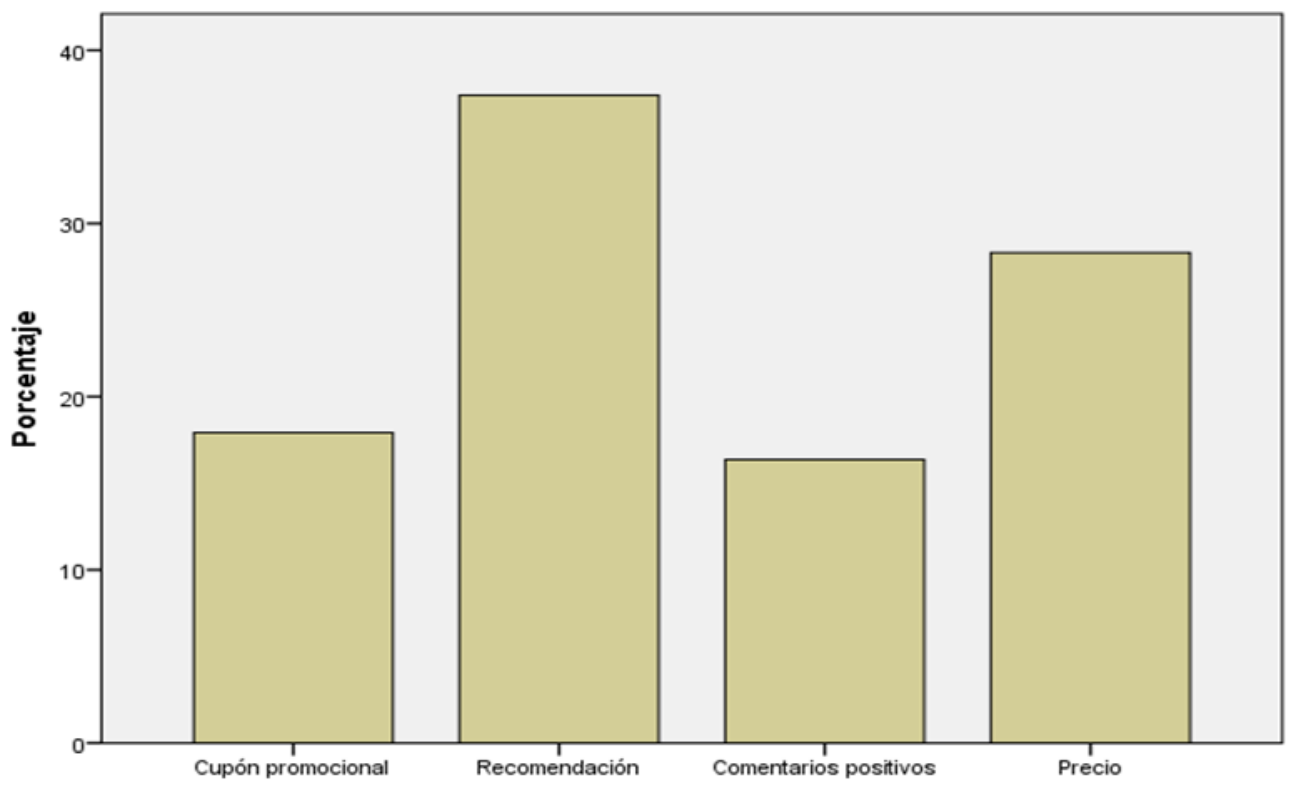

Elaborado por: Autores (2018)

De acuerdo a la encuesta realizada a los 9 cantones de la provincia de Tungurahua sobre los aspectos ante un anuncio publicitario sobresale con un máximo porcentaje la recomendación es de $37.4 \%$, con un $28.3 \%$ precio, cupón promocional con un $17.9 \%$ contenido de la publicidad y como un porcentaje $16.4 \%$ menor existe los comentarios positivos.

Tabla 3 Análisis chi cuadrado

\begin{tabular}{lcccccc}
\hline & ANÁLISIS CHI-CUADRADO & & & \\
& & & & & \\
& PRODUCTO & MERCADO & PUBLICIDAD & ANUNCIO & COMPRA \\
\hline OFERTAS & 6.323 & 6.170 & 3.626 & 2.766 & 22.918 \\
DECISIÓN FINAL DE COMPRA & 26.258 & 15.163 & 25.676 & 55.967 & 42.050 \\
SERVICIO & 18.819 & 7.959 & 13.098 & 8.161 & 18.272 \\
DECISIÓN DE COMPRA & 17.261 & 6.884 & 12.511 & 12.328 & 16.512 \\
REDES SOCIALES & 14.832 & 7.029 & 10.835 & 9.179 & 24.101 \\
\hline
\end{tabular}

Elaborado por: Autores (2018) 
De acuerdo al análisis que nos brinda la tabla del chi cuadrado se puede dar a conocer que las variables se encuentran asociadas entre ellas de esta manera las empresas comerciales buscan mantenerse dentro del mercado, crecer a nivel nacional e internacional y fomentar fidelización con clientes potenciales.

Tabla 4 Valor $P$ en chi cuadrado

\begin{tabular}{lccccc}
\hline \multicolumn{7}{c}{ VALOR P EN CHI CUADRADO } \\
\hline & PRODUCTO & MERCADO & PUBLICIDAD & ANUNCIO & COMPRA \\
OFERTAS & 0.707 & 0.723 & 0.934 & 0.973 & $\mathbf{0 . 0 0 6}$ \\
DECISIÓN FINAL & $\mathbf{0 . 0 0 2}$ & 0.087 & $\mathbf{0 . 0 0 2}$ & $\mathbf{0 . 0 0 0}$ & $\mathbf{0 . 0 0 0}$ \\
SERVICIO & $\mathbf{0 . 0 2 7}$ & 0.538 & 0.158 & 0.518 & $\mathbf{0 . 0 3 2}$ \\
DECISIÓN DE COMPRA & $\mathbf{0 . 0 4 5}$ & 0.649 & 0.186 & 0.195 & 0.057 \\
REDES SOCIALES & 0.096 & 0.634 & 0.287 & 0.421 & $\mathbf{0 . 0 0 4}$ \\
\hline
\end{tabular}

\section{Elaborado por: Autores (2018)}

En la tabla 4 sobre el valor P en chi cuadrado se podría analizar que la mayoría de las variables no están relacionadas ya que el valor se encuentra elevado a lo determinado, el valor máximo es de 0.005 por lo que al observar la tabla da a conocer que el producto se encuentra relacionado con la decisión final de compra con un 0.002; al igual que con el servicio con un 0.027 y con la decisión de compra con un 0.045 ; la publicidad y el anuncio se relaciona con la decisión final de compra con un 0.002 por este motivo dichas empresas optan por realizar publicidad para llamar la atención del público. En si la compra es otra de las variables que tienen relación con las ofertas con un 0.006, decisión final de compra 0.000 , servicio 0.0032 y redes sociales 0.004 , muchas de estas permiten que las empresas fomenten distintas estrategias para dar a conocer de forma eficiente sus productos.

\section{Discusión}

Se permitió validar, las demás investigaciones evaluándose, las medidas en como los usuarios, hacen la elección posterior para adquirir sus productos, midiéndose cuanto es el impacto de la publicidad en ellos, y la manera en como se refleja en sus compras, es por ello que este apartado hace especial mención en cuanto, a las gráficas ya analizadas anteriormente, la primera pregunta hizo énfasis de manera inicial, en cuanto a los aspectos necesarios, en que la publicidad pueda 
llegar ante la percepción de los usuarios, ya que en su mayoría, los encuestados afirman que la creatividad influye altamente dentro del nivel en la publicidad, ya que al encontrar nuevas estrategias, el consumidor final se siente ansioso por conocer y poder adquirir, en primera instancia el producto.

Según lo manifestado anteriormente, los encuestados afirmaron en segundo lugar que los estímulos sensoriales, son importantes al momento de, que se observa la publicidad, pues en casos suelen impactar las emociones, según investigaciones mencionadas. Cabe citar la de (Jenny Camino, 2014), ya que se basó en la búsqueda y estrategias, necesarias para que el producto de una empresa pueda venderse, obteniendo como resultado, que era indispensable generar el impacto de la publicidad para poder aumentar el número de ventas, y así posicionarse en las elecciones de sus usuarios, confirmando con ello la encuesta hecha hacia los usuarios de diferentes productos comercializado.

Las decisiones de compras supieron manifestar que una mayoría significante prefieren precios accesibles, y en segundo lugar expresaron que prefieren la recomendación de amigos, y familiares, en la toma de decisiones para la compra de los productos, de acuerdo a la investigación de (Abraham Geifman, 2014), acerca de la decisión de compra, define que lo que realmente vende, es que en su aviso publicitario indica influye realmente es demostrar la necesidad que tiene del producto, observando que los avisos publicitarios en la provincia de Tungurahua, son bajos en contenido del marketing online y el marketing offline.

\section{Conclusiones}

El impacto publicitario es un mecanismo necesario para poder, predominar en la decisión de los usuarios para la compra, por lo tanto, con una publicidad baja por parte de las empresas comerciales, se está hablando de que se evidencian pocas y vendas, y en resumidas palabras no hay lanzamientos de nuevas marcas, y productos, mismos que son necesitados por los usuarios, necesidades que aún no han sido compensadas.

En si los mercadólogos deben interesarse por poner a la disposición y a la mano del consumidor toda la información de sus productos en todos los medios posibles, puesto que los consumidores pueden emprender la búsqueda de información por ciertos medios y que efectivo seria que en 
todos los medios que el consumidor consulte se frecuente nuestra marca siendo así se tendría mayores posibilidades de ser seleccionado por el consumidor.

\section{Referencias Bibliográficas}

Abraham Geifman. (2014). Forbes. Obtenido de todas las decisiones de compras son emocionales: https://www.forbes.com.mx/todas-las-decisiones-de-compra-son-emocionales/ American Marketing Asociation. (2016). La publicidad. Obtenido de http://uachatec.com.mx/wpcontent/uploads/2016/08/0.-

Publicidad_y_Promocion_Integral_de_Marca_O_Guinn_Thomas_.pdf

Aravela \& Cols. (2006). La Encuesta. Obtenido de http://uvsfajardo.sld.cu/diferencia-entrecuestionario-y-encuesta

Bello, Vázquez y Trespalacios. (2016). Objetivos de la comunicación publicitaria. Obtenido de file://C:/Users/Usuario/Downloads/203_00.pdf

Bill Berbanch. (2013). La publicidad. Obtenido de file://C:/Users/Usuario/Downloads/203_00.pdf

Deming, E. (2018). Calidad del producto. Obtenido de http://www.amamex.org.mx/articulos/Los-14-Puntos-sobre-Calidad-de-Edwards-Deming.html

Doris Martínez. (2016). FACTORES CLAVE EN MARKETING ENFOQUE EMPRESAS DE SERVICIOS. Obtenido de http://www.redalyc.org/pdf/709/70946593003.pdf

González. (2014). Discordancias entre las organizaciones empresariales. REDALYC. Obtenido de http://www.redalyc.org/pdf/709/70946593003.pdf

Grinnell. (2006). Paradigma de la Investigación. México: 4ta Edición Metodologia de la investigación. 
Jenny Camino. (2014). Estrategias de publicidad y su impacto en las ventas de la Empresa Repremarva de la ciudad de Ambato, durante el año 2014. Obtenido de http://repositorio.uta.edu.ec/bitstream/123456789/7585/1/141\%20MKT.pdf

Juran y $\quad$ Gryna. (2010). La calidad. Obtenido de http://xxxturismoxxx.blogspot.com/2010/03/conceptos-de-calidad-segun-diversos.html

Kotler, Armstrong. (2018). Marketing Teacher. Obtenido de https://www.ukessays.com/dissertation/examples/marketing/purchasing-decision-makingprocess.php

Luis Bassat. (2016). EL LIBRO ROJO DE LA PUBLICIDAD. En L. Bassat. Obtenido de https://www.pqs.pe/sites/default/files/2016/03/aprende-mas/libro_rojo_de_la_publicidad1.pdf

María Tobar. (2013). La toma de decisiones en el mundo del consumo basado en procesos cerebrales. Obtenido de http://repositorio.usfq.edu.ec/bitstream/23000/2158/1/106124.pdf

\section{Moreno, E. (2013). INSTRUMENTOS DE INVESTIGACIÓN.}

O’Guinn, Allen y Semenik. (2016). Promonegocios. Obtenido de http://uachatec.com.mx/wpcontent/uploads/2016/08/0.-

Publicidad_y_Promocion_Integral_de_Marca_O_Guinn_Thomas_.pdf

Olsztyn Mario. (2018). Consultoria \& Capacitación en Comportamiento Organizacional \& Gestión Estratégica. Obtenido de https://www.marioolsztyn.com/articulos/74-el-proceso-de- compra.html

Pablo Arcos. (2014). ANÁLISIS DEL COMPORTAMIENTO DEL CONSUMIDOR EN LA COMPRA DE TABLETS EN LA CIUDAD DE QUITO, PARA PERSONAS ENTRE LOS 18 A 40 AÑOS DE EDAD, EN EL AÑO 2013. Obtenido de http://repositorio.puce.edu.ec/bitstream/handle/22000/7816/2.22.000818.pdf;sequence=4

Philip Kotler. (2013). DEFINICIÓN DE PUBLICIDAD. Obtenido de file://C:/Users/Usuario/Downloads/203_00.pdf 
Philip Kotler. (2013). Marketing punto de venta. Obtenido de https://www.promotienda.es/5fases-en-el-proceso-de-decision-de-compra-segun-kotler/

Pobea, M. (2015). LA ENCUESTA. Obtenido de http://files.sld.cu/bmn/files/2015/01/laencuesta.pdf

Rojas, I. (2011). ELEMENTOS PARA EL DISEÑO DE TÉCNICAS DE INVESTIGACIÓN:. Redalyc, 277-297.

Schultz, Martin y Brown. (2014). Enfoques de los objetivos de una campaña publicitaria. Obtenido de https://ddd.uab.cat/pub/tfg/2014/126242/TFG_Jennifer_Rodriguez_Ponce.pdf Sonia Ottaviano. (2015). ANÁLISIS DEL IMPACTO DE LA PUBLICIDAD EN LAS DECISIONES DE CONSUMO EN LOS NIÑOS.

Tamayo, M. (2016). TIPOS DE INVESTIGACIÓN. Obtenido de https://trabajodegradoucm.weebly.com/uploads/1/9/0/9/19098589/tipos_de_investigacion.pdf Webloyalty y Oxford Brookes University. (2017). Solo marketing. Obtenido de https://solomarketing.es/5-motivos-que-llevan-a-los-consumidores-a-tomar-la-decision-decompra/ 